\title{
Scaling of gain bounds for switched adaptive control with large uncertainties
}

\author{
Dominic Buchstaller and Mark French
}

\begin{abstract}
A wide class of MMAC with a finite $l_{p}(1 \leq$ $p \leq \infty)$ closed loop gain are shown to have an unboundedly increasing $l_{p}$ closed loop gain for a simple set of plants under increasing parametric uncertainty. A modification is proposed which achieves a quadratic closed loop gain function which is independent of the size of the uncertainty set.
\end{abstract}

\section{INTRODUCTION}

It is well known that the gain from external input and output disturbances to the internal signals of a closed loop system, containing a plant $P$ and a controller $C$, is a key determinant of the robustness of the resulting system. For example for linear systems, the reciprocal of the $l_{2}$ gain of the operator mapping input and output disturbances to the input and the output of the plant is called the robust stability margin $b_{P, C}$ : all plants $P_{1}$ which are within the open gap ball of size $b_{P, C}$, centred at $P$, are also stabilized by $C$. Similar statements hold in the nonlinear setting [7].

This motivates the need to examine the behaviour of such a closed loop gain for multiple model adaptive control (MMAC). Here we consider a class of MMAC and first show that the resulting $l_{p}$ closed loop gain (studied in [6]), whilst finite for any fixed uncertainty level, nevertheless scales poorly as the parametric uncertainty of the system increases. We expect that similar examples can be constructed for most MMAC schemes, and interpret this is an indication that the standard $l_{p}$ gain is an inappropriate measure of stability, see also [3],[10].

In the light of this fundamental example, we examine a gain function (actually a quadratic) measure of the gain of the closed loop operator and show that this gain function is dependent on the actual plant, and independent of the uncertainty level of the system; thus demonstrating good scaling properties in the face of an appropriate measure of stability. By comparison, we observe the closed loop gain function analysis of the more classical parametric adaptive controllers in [4],[5] also yield gain functions with superlinear growth; this may be an intrinsic feature of a nonconservative analysis on a large uncertainty set. Furthermore, robustness certificates can also be given for controllers with such properties [4],[5],[7].

The class of MMAC algorithms under consideration observes in- and output signals of a process and explains this "observation" by disturbances acting on candidate plant

D. Buchstaller is with the School of Electronics and Computer Science, University of Southampton, SO17 1BJ, UK, dpb05rdecs.soton.ac.uk

M. French is with the School of Electronics and Computer Science, University of Southampton, SO17 1BJ, UK, mcfeecs. soton . ac . uk models in a finite plant model set. At every time step the controller corresponding to the plant model which is able to "explain" the observation by the smallest disturbance signal is chosen. This approach was introduced in [2] and [10] where gain bounds were obtained for the case of two first order plants. A more general gain bound analysis was developed in [6], which provides the basis for the development of the results in this paper. Gain bound analysis is also presented (in continuous time) in various results by Morse et al. e.g. [9] for switched controllers based on observer estimators.

The analysis is performed over the same class of systems as in [6], i.e. we assume all plants to be of relative degree one and the underlying controllers to be dead beat. Such gain function results allow us to establish semi-global robustness guarantees in the sense of the nonlinear gap metric, see [4], [7].

Finally we remark that the restrictive requirement that the controllers are dead beat is made for simplicity, the generalization of this is a topic of current research.

\section{DEFINITIONS}

\section{A. Norms and signals}

Let

$$
\mathcal{S}=\operatorname{map}\left(\mathbb{N}, \mathbb{R}^{h}\right), h \in \mathbb{N} .
$$

Let a signal $a \in S$ be in $l_{p}, 1 \leq p \leq \infty$ iff

$$
\begin{aligned}
& l_{p}:=\left\{\left.a \in \mathcal{S}\left|\sum_{0 \leq i<\infty}\right| a_{i}\right|^{p}<\infty\right\}, \\
& l_{\infty}:=\left\{a \in \mathcal{S}|| a_{i} \mid<\infty, 0 \leq i<\infty\right\} .
\end{aligned}
$$

Let the spaces be equipped with the corresponding norms

$$
\|a\|_{p}:=\left(\sum_{0 \leq i<\infty}\left|a_{i}\right|^{p}\right)^{1 / p},\|a\|_{\infty}:=\sup _{0 \leq i<\infty}\left|a_{i}\right| .
$$

Given a signal space $\mathcal{V}$, define the "extended" signal space

$$
\mathcal{V}_{e}:=\left\{a \in \mathcal{S} \mid \forall k \in \mathbb{N}: \mathscr{T}_{k} a \in \mathcal{V}\right\}
$$

where for $v \in S, K \in \mathbb{N}$ the truncation operator $\mathscr{T}_{K}: \mathcal{S} \rightarrow \mathcal{S}$ is defined by

$$
\mathscr{T}_{K} v(k)=\left\{\begin{array}{cc}
v(k), & 0 \leq k \leq K \\
0, & \text { otherwise }
\end{array}\right.
$$

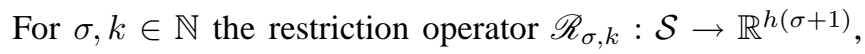
$h \in \mathbb{N}$ is defined by

$$
\mathscr{R}_{\sigma, k} v(i):=v(k-\sigma+i), 0 \leq i \leq \sigma .
$$

An operator $O: \mathcal{S} \rightarrow \mathcal{S}$ is said to be to be causal iff

$$
\mathscr{T}_{k} O \mathscr{T}_{k} v=\mathscr{T}_{k} O v, \forall k \in \mathbb{N}, v \in \mathcal{S} .
$$




\section{B. Plant and controller}

Let $\mathcal{U}=\mathcal{Y}=l_{p}, 1 \leq p \leq \infty$. Given a plant $P: \mathcal{U}_{e} \rightarrow \mathcal{Y}_{e}$ and a controller $C: \mathcal{Y}_{e} \rightarrow \overline{\mathcal{U}}_{e}$ the closed loop system $[P, C]$ under consideration in figure 1 is defined via the following set of system equations:

$$
\begin{aligned}
y_{1} & =P u_{1}, u_{0}=u_{1}+u_{2}, y_{0}=y_{1}+y_{2}, \\
u_{2} & =C y_{2}
\end{aligned}
$$

with $u_{0} \in \mathcal{U}, y_{0} \in \mathcal{Y}, u_{1}, u_{2} \in \mathcal{U}_{e}, y_{1}, y_{2} \in \mathcal{Y}_{e}$ where $\left(u_{0}, y_{0}\right)$ represent the input and output disturbances, $\left(u_{1}, y_{1}\right)$ represents the plant input and output and $\left(u_{2}, y_{2}\right)$ represents the observed signal or observation.

Let $\sigma>0 \in \mathbb{N}$, and as in [6] let the class of plants under

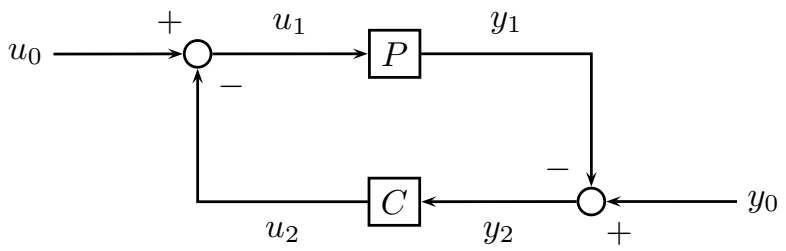

Fig. 1. The closed loop system $[P, C]$

consideration be specified by:

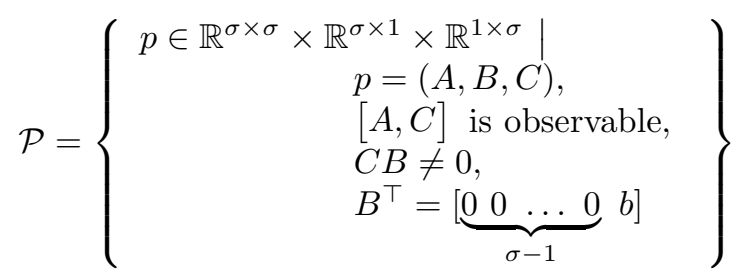

where $b \in \mathbb{R}$.

For all $p=(A, B, C) \in \mathcal{P}$ define the plant operator $P_{p}$ by

$$
P_{p}: \mathcal{U}_{e} \rightarrow \mathcal{Y}_{e}: u_{1} \mapsto y_{1}
$$

with

$$
\begin{aligned}
x(k+1) & =A x(k)+B u_{1}(k) \\
y_{1}(k) & =C x(k) \\
x(-k) & =0 \quad \forall k \in \mathbb{N} .
\end{aligned}
$$

It is well known that for all $p \in \mathcal{P}, P_{p}$ is stabilizable by a unique controller $C_{p}$ of the form:

$$
C_{p}: u_{2}(k)=-a_{p}^{\top}\left[\begin{array}{c}
y_{2}(k-\sigma+1) \\
\vdots \\
y_{2}(k)
\end{array}\right]
$$

with the dead beat property, i.e. such that for all $k \in \mathbb{N}$ and for all $\left(u_{0}, y_{0}\right) \in \mathcal{U} \times \mathcal{Y}$ the closed loop $\left[P_{p}, C_{p}\right]$ satisfies:

$$
\left.u_{0}\right|_{(k, \infty)}=\left.y_{0}\right|_{(k, \infty)}=\left.0 \Rightarrow y_{1}\right|_{[k+1, \infty)}=0 .
$$

Hereafter we restrict our attention to such controllers.

\section{Robustness and the closed loop operator}

Let $\mathcal{W}=\mathcal{U} \times \mathcal{Y}, \mathcal{W}_{e}=\mathcal{U}_{e} \times \mathcal{Y}_{e}, w_{i}=\left(u_{i}, y_{i}\right), i=0,1,2$. To study the effects of input disturbances on the closed loop signals it is useful to have some notion of system gain. For that purpose we introduce the closed-loop operator

$$
\Pi_{P / / C}: \mathcal{W} \rightarrow \mathcal{W}_{e}: w_{0} \rightarrow w_{1},
$$

and we define a system $[P, C]$ to be (BIBO) gain stable if $\Pi_{P / / C}$ is gain stable, i.e. $\exists M>0$ s.t.

$$
\left\|\Pi_{P / / C}\right\| \leq M \text {. }
$$

\section{The switched control system}

Given a set of candidate plants, with a corresponding set of candidate dead beat controllers, the basic MMAC algorithm can informally be described as follows. At each time step, and for each candidate plant, the size of the smallest disturbance up to the current time which is consistent with the observation is calculated and the controller corresponding to the plant with the smallest such disturbance 'explanation' is switched into closed loop.

We first need to define for which plants we want to invoke disturbance estimation. For that purpose we introduce the parameter set $\mathcal{P}_{i}$ corresponding to the set of plants we want to consider for disturbance estimation. Further analysis will show that it is advantageous to define this set as time varying and dependent on the observation.

Let the set of plants under consideration with $i \in \mathbb{N}$ be given by

$$
\begin{aligned}
\mathcal{P}_{i}: & =\left\{p_{1}, p_{2}, \ldots, p_{i}\right\} \\
& =\left\{\left(A_{1}, B_{1}, C_{1}\right),\left(A_{2}, B_{2}, C_{2}\right), \ldots,\left(A_{i}, B_{i}, C_{i}\right)\right\} .
\end{aligned}
$$

where

$$
\mathcal{P}_{1} \subseteq \mathcal{P}_{2} \subseteq \cdots \subseteq \mathcal{P} .
$$

Let $\Omega=\left\{\mathcal{P}_{i} \mid i \in \mathbb{N}\right\}$. Let $G$ denote a "plant generating" operator

$$
G: \mathcal{W}_{e} \rightarrow \operatorname{map}(\mathbb{N}, \Omega)
$$

subject to the constraint

$$
G\left(w_{2}\right)(0)=\mathcal{P}_{1}, G\left(w_{2}\right)(k) \subseteq G\left(w_{2}\right)(k+1) \forall k \in \mathbb{N} .
$$

A trivial but important example of a plant generating operator is the constant mapping

$$
G\left(w_{2}\right)(k)=P^{*} \subseteq \mathcal{P},
$$

however it is advantageous to allow this operator to take a more complex form, as will be shown in section 4 .

Formally the causal switching operator

$$
S: \mathcal{W}_{e} \rightarrow \operatorname{map}(\mathbb{N}, \Omega): w_{2} \mapsto q
$$

is given by the combination of the estimation operator $E$, the norm operator $N$, the minimization operator $M$ and the plant generating operator $G$ with

$$
S=M(N E, G)
$$

where $M, N, E$ are defined below. 
Let the time series of disturbance estimates up to time $k$ corresponding to a plant $p \in \mathcal{P}$ be given by

$$
d_{p}[k]: \mathbb{N} \rightarrow \operatorname{map}\left(\mathbb{N}, \mathbb{R}^{h}\right)
$$

and

$$
d_{p}[k]=\left(d_{p}[k](0), d_{p}[k](1), \ldots, d_{p}[k](k), 0, \cdots\right)
$$

with $h \in \mathbb{N}$ being dependent on the utilized estimator as well as the plant order $\sigma \in \mathbb{N}$. We now define for $k \in \mathbb{N}, p \in \mathcal{P}$ the estimation operator

$$
E: \mathcal{W}_{e} \rightarrow \operatorname{map}\left(\mathbb{N}, \operatorname{map}\left(\mathcal{P}, \operatorname{map}\left(\mathbb{N}, \mathbb{R}^{h}\right)\right)\right)
$$

by

$$
w_{2} \mapsto\left[k \mapsto\left(p \mapsto d_{p}[k]\right)\right] ;
$$

the norm operator

$$
N: \operatorname{map}\left(\mathbb{N}, \operatorname{map}\left(\mathcal{P}, \operatorname{map}\left(\mathbb{N}, \mathbb{R}^{h}\right)\right)\right) \rightarrow \operatorname{map}\left(\mathbb{N}, \operatorname{map}\left(\mathcal{P}, \mathbb{R}^{+}\right)\right)
$$

by

$$
\left[k \mapsto\left(p \mapsto d_{p}[k]\right)\right] \mapsto\left[k \mapsto\left(p \mapsto\left\|d_{p}[k]\right\|\right)\right) ;
$$

and the minimization operator

$$
M:\left(\operatorname{map}\left(\mathbb{N}, \operatorname{map}\left(\mathcal{P}, \mathbb{R}^{+}\right)\right), \operatorname{map}(\mathbb{N}, \Omega)\right) \rightarrow \operatorname{map}(\mathbb{N}, \mathcal{P})
$$

by

$$
\left[k \mapsto\left(p \mapsto r_{p}[k]\right),\left(k \mapsto \mathcal{P}_{i(k)}\right)\right] \mapsto[k \mapsto q(k)]
$$

where

$$
q(k)=\operatorname{argmin}_{\mathrm{p} \in \mathcal{P}_{\mathrm{i}(\mathrm{k})}} \mathrm{r}_{\mathrm{p}}[\mathrm{k}],
$$

and we assume that $\operatorname{argmin}_{\mathrm{p} \in \mathcal{P}_{\mathrm{i}(\mathrm{k})}}, \mathrm{i}(\mathrm{k}) \in \mathbb{N}$ returns the parameter $p_{j}$ corresponding to the smallest index $j \in \mathbb{N}$ if there exist multiple minimal $r_{p}[k], p \in \mathcal{P}_{i}$. Hence $S$ defines the map

$$
q(k)=\operatorname{argmin}_{\mathrm{p} \in \mathrm{G}\left(\mathrm{w}_{2}\right)(\mathrm{k})}\left\|\mathrm{d}_{\mathrm{p}}[\mathrm{k}]\right\| .
$$

Finally let the switching controller

$$
C: \mathcal{Y}_{e} \rightarrow \mathcal{U}_{e}: y_{2} \mapsto u_{2}
$$

be defined from equation (2.8) by

$$
\begin{aligned}
\left(C y_{2}\right)(k) & =\left(C_{q(k)} y_{2}\right)(k) \\
& =-\left(a_{q(k)}^{\top} \mathscr{R}_{\sigma-1, k} y_{2}\right)(k) .
\end{aligned}
$$

\section{E. Properties of the estimator}

We define the set $W_{p}(k)$ of weakly consistent disturbance signals to a plant $p \in \mathcal{P}$ and the observation $\left(u_{2}, y_{2}\right)$ to be

$$
W_{p}(k):=\left\{\begin{aligned}
& v \in \mathbb{R}^{\sigma} \times \mathbb{R}^{\sigma+1} \mid \\
& \exists\left(u_{0}^{p}, y_{0}^{p}\right) \in \mathcal{W}_{e} \text { s.t. } \\
& \mathscr{R}_{\sigma, k} P_{p}\left(u_{0}^{p}-u_{2}\right)=\mathscr{R}_{\sigma, k}\left(y_{0}^{p}-y_{2}\right) \\
& v=\left(\mathscr{R}_{\sigma-1, k-1} u_{0}^{p}, \mathscr{R}_{\sigma, k} y_{0}^{p}\right)
\end{aligned}\right\}
$$

and we consider a vector $v \in \mathbb{R}^{\sigma} \times \mathbb{R}^{\sigma+1}$ to be weakly consistent with $\left(u_{2}, y_{2}\right)$ and the plant $p$ iff $v \in W_{p}(k)$.

Instead of now giving an explicit algorithm for the "disturbance estimator" $N E$ we state some general assumptions.
Let $p_{*}$ be the parameter corresponding to the "true" unknown plant $P:=P_{p_{*}} \in \mathcal{P}$.

\section{Assumption 2.1:}

1) (Causality): $N E$ is causal. Note that $N$ is always causal and therefore it suffices to assume that $E$ is causal.

2) (Minimality): There exists a $c_{1}>0$ such that for all $k \geq 0$ and for all $w_{2} \in \mathcal{W}_{e}, w_{0} \in \mathcal{W}$ satisfying equation (2.1) for $P=P_{p_{*}}$

$$
N E\left(w_{2}\right)(k)\left(p_{*}\right)=\|\left(E\left(w_{2}\right)(k)\left(p_{*}\right)\left\|\leq c_{1}\right\| w_{0} \| .\right.
$$

3) (Weak consistency): There exists a map

$$
\Phi: \operatorname{map}\left(\mathbb{N}, \mathbb{R}^{h}\right) \rightarrow \mathbb{R}^{\sigma} \times \mathbb{R}^{\sigma+1},
$$

such that for all $w_{2} \in \mathcal{W}_{e}, w_{0} \in \mathcal{W}, p \in \mathcal{P}$ satisfying equation (2.1) for $P=P_{p}$

$$
\Phi E\left(w_{2}\right)(k)(p) \in W_{p}(k)
$$

and

$$
\left\|\Phi E\left(w_{2}\right)(k)(p)\right\| \leq\left\|\mathscr{R}_{\sigma, k} E\left(w_{2}\right)(k)(p)\right\| .
$$

4) (Monotonicity): For all $p \in \mathcal{P}$, for all $k, l \in \mathbb{N}$ with $0 \leq k \leq l$ and for all $w_{2} \in \mathcal{W}_{e}, w_{0} \in \mathcal{W}$ satisfying equation (2.1) for $P=P_{p}$ there holds

$$
\left\|E\left(w_{2}\right)(k)(p)\right\| \leq\left\|\mathscr{T}_{k} E\left(w_{2}\right)(l)(p)\right\| .
$$

By assumption 2.1(4), consistency of the disturbance estimates is only required over the last $\sigma$ steps and therefore finite horizon estimation is sufficient. In [6] a finite and an infinite horizon estimator are presented fullfilling the above assumptions.

Also note that it is sufficient to determine $N E$ without the explicit construction of $E$ as long as the disturbance estimator fullfills the given assumption on it's disturbance estimates, e.g. the Kalman filter can be used for this purpose in $l_{2}$, as discussed in [2],[10].

\section{A MMAC ALGORITHM WHICH DOES NOT SCALE}

We will now show that the standard version of the algorithm, with a constant plant generating operator $G$, can produce large closed loop gains for large parameter uncertainties. Since this is a negative result we demonstrate the effect on a simple example.

Let the true unknown plant $P=P_{p_{*}}$ be given by

$$
p^{*}=\left(i_{*}, 1,1\right), i_{*} \in \mathbb{N}
$$

where we assume $1 \leq i_{*} \leq l \in \mathbb{N}$ and the uncertainty level $l=$ const. $\in \mathbb{N}$ to be known. Hence we let the set of plants under consideration be parametrized by the uncertainty level $l \geq 1$ and be given by:

$$
\mathcal{P}_{l}=\{(1,1,1),(2,1,1), \ldots,(l, 1,1)\}
$$

and let

$$
G\left(w_{2}\right)(k)=\mathcal{P}_{l}, \quad \forall k \in \mathbb{N} \text {. }
$$


The corresponding dead-beat controller $C_{p}: \mathcal{Y}_{e} \rightarrow \mathcal{U}_{e}$ to each $p \in \mathcal{P}_{l}$ is defined by

$$
C_{p}: u_{2}=-i y_{2}, \forall p=(i, 1,1) \in \mathcal{P}_{l} .
$$

Observe that for all $p \in \mathcal{P}_{l},\left[P_{p}, C_{p}\right]$ is gain stable. The switching controller is given by

$$
C\left[\mathcal{P}_{l}\right]: \mathcal{Y}_{e} \rightarrow \mathcal{U}_{e}: y_{2} \mapsto u_{2}
$$

with

$$
\left(C\left[\mathcal{P}_{l}\right] y_{2}\right)(k):=\left(C_{q(k)} y_{2}\right)(k),
$$

where $q(k)$ is determined by equations (2.11)-(2.19) and equation (3.22) where for $p \in \mathcal{P}_{l}$ the estimation operator $E$ from equations (2.15),(2.16) is explicitly defined by

$$
d_{p}[k]:=\operatorname{argmin}_{\mathrm{X} \in \mathrm{S}_{\mathrm{p}}(\mathrm{k})}\{\|\mathrm{X}\|\}
$$

and

$$
\begin{aligned}
& S_{p}(k):=\left\{\left(\mathscr{T}_{k-1} u_{0}^{p}, \mathscr{T}_{k} y_{0}^{p}\right) \in \mathcal{W} \mid\right. \\
& \left.\mathscr{T}_{k} P_{p}\left(\mathscr{T}_{k-1} u_{0}^{p}-\mathscr{T}_{k-1} u_{2}\right)=\mathscr{T}_{k} y_{0}^{p}-\mathscr{T}_{k} y_{2}\right\}
\end{aligned}
$$

Theorem 3.1: The given estimator fullfills assumption 2.1. Proof The proof can be found in [6] and is omitted. From [6] we know that:

$$
\left\|\Pi_{P / / C\left[\mathcal{P}_{l}\right]}\right\|<\infty, \forall l>0,
$$

however the gain bound scales poorly with $l$, as we show next.

Theorem 3.2: Let $l>0$ and let the parameter set $\mathcal{P}_{l}$ be given by equation (3.21). Let the switching operator $S$ be defined by equations (2.11)-(2.19). Let the switching controller $C\left[\mathcal{P}_{l}\right]$ be given by equations (3.24),(3.25). Then for $p_{*}=(1,1,1), P=P_{p_{*}}$ the closed loop system $\left[P, C\left[\mathcal{P}_{l}\right]\right]$ has the property that there does not exist an $M>0$ such that

$$
\left\|\Pi_{P, C\left[\mathcal{P}_{l}\right]}\right\| \leq M, \forall l>0 .
$$

Proof The proof is in two steps. Firstly, we show that we can always make the switching algorithm switch to the controller corresponding to the plant with the largest possible $a \in \mathbb{N},(a, 1,1) \in \mathcal{P}_{l}$, that is $a=l \in \mathbb{N}$. Secondly, we show that this condition leads to the unbound increase of the gain of the closed loop operator as $l$ increases.

Let $p_{b}=(b, 1,1), p_{l}=(l, 1,1) \in \mathcal{P}_{l}, 1 \leq b<l$. Now consider the closed-loop system $\left[P, C\left[\mathcal{P}_{l}\right]\right]$ and let

$$
\left(\begin{array}{l}
u_{0} \\
y_{0}
\end{array}\right)=\left(\left(\begin{array}{l}
0 \\
B
\end{array}\right),\left(\begin{array}{l}
0 \\
0
\end{array}\right),\left(\begin{array}{c}
0 \\
B-l B
\end{array}\right),\left(\begin{array}{l}
0 \\
0
\end{array}\right),\left(\begin{array}{l}
0 \\
0
\end{array}\right), \cdots\right)
$$

where $B>0$. We now claim that these disturbances make the algorithm switch to the controller $C_{p_{l}}$ in two time steps, i.e. $q(2)=p_{l}$ and that the signals in table I are consistent with the closed loop $\left[P, C\left[\mathcal{P}_{l}\right]\right]$, where throughout this proof, a vector with an entry marked $\times$ indicates that the entry is irrelevant to the calculation that follows.

To see this we argue as follows. Let $u_{0}, y_{0}$ be as in table I. At time $k=0$ the disturbance estimates $d_{p}[0], p \in\left\{p_{b}, p_{l}\right\}$ are forced by $y_{0}(0)=B$ and zero initial conditions to be

$$
d_{p}[0]=\left(\begin{array}{c}
\times \\
y_{0}^{p}(0)
\end{array}\right)=\left(\begin{array}{c}
\times \\
B
\end{array}\right), p \in\left\{p_{b}, p_{l}\right\} .
$$

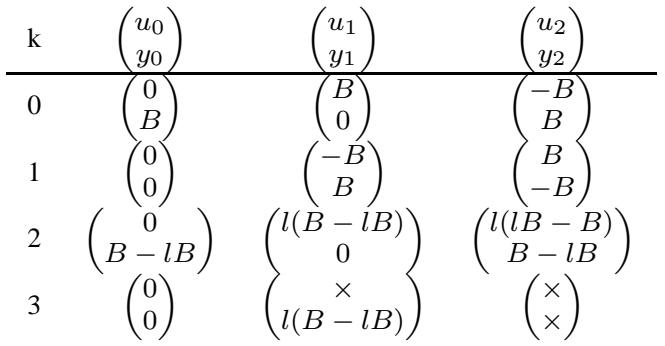

TABLE I

Signals For $\left[P, C\left[\mathcal{P}_{l}\right]\right]$ UP TO TIME $k=3$

Consequently $\left\|d_{p}[0]\right\|=B, p \in\left\{p_{b}, p_{l}\right\}$ and $q(0)=p_{*}$. It is trivial to verify that the entries in table $I$ are consistent with equations (2.1),(2.2) for $P=P_{1}, C=C\left[\mathcal{P}_{l}\right]$ and $k=0$.

At time $k=1$ we have $y_{1}(1)=B$ and with $y_{0}(1)=0$ there holds $y_{2}(1)=-B$. By assumption 2.1(4), that is $\left\|d_{p}[k]\right\| \leq\left\|d_{p}[k+1]\right\|, p \in \mathcal{P}, k \in \mathbb{N}$ the smallest disturbance $d_{p}[1], p \in\left\{p_{b}, p_{l}\right\}$ consistent with $\left(\mathscr{T}_{0} u_{2}, \mathscr{T}_{1} y_{2}\right)$ and $P_{p_{b}}, P_{p_{l}}$ can be found to be

$$
d_{p}[1]=\left(\left(\begin{array}{c}
0 \\
B
\end{array}\right)\left(\begin{array}{c}
\times \\
0
\end{array}\right)\right), p \in\left\{p_{b}, p_{l}\right\} .
$$

Since $\left\|d_{p_{l}}[1]\right\|=\left\|d_{p_{b}}[1]\right\|, q(1)=p_{*}$ and no switch occurs. It is trivial to verify that the entries in table $I$ are consistent with equations (2.1),(2.2) for $P=P_{1}, C=C\left[\mathcal{P}_{l}\right]$ and $k=1$.

At time $k=2$ we have $y_{1}(2)=0$ and with $y_{0}(2)=B-l B$ it follows that $y_{2}(2)=B-l B$. Now the smallest disturbance estimate for $d_{p_{l}}[2]$ consistent with $\left(\mathscr{T}_{1} u_{2}, \mathscr{T}_{2} y_{2}\right)$ and $P_{p_{l}}$ is

$$
d_{p_{l}}[2]=\left(\left(\begin{array}{l}
0 \\
B
\end{array}\right)\left(\begin{array}{l}
0 \\
0
\end{array}\right)\left(\begin{array}{l}
\times \\
0
\end{array}\right)\right)
$$

since similarly minimality is ensured by consistency and $\left\|d_{p_{l}}[2]\right\|=\left\|d_{p_{l}}[1]\right\|$. In fact the disturbances $\left(u_{0}, y_{0}\right)$ are not arbitrary but have been chosen so that this holds.

Since $y_{0}^{p_{b}}(0)=B,\left\|d_{p_{b}}[2]\right\| \geq\left\|d_{p_{l}}[2]\right\|$, however $d_{p_{b}}[2]=$ $d_{p_{l}}[2], p_{b} \neq p_{l}$ is not possible since the trajectories would have the property that

$$
\Pi_{C\left[\mathcal{P}_{l}\right] / / P_{p_{b}}} d_{p_{b}}[2]=\Pi_{C\left[\mathcal{P}_{l}\right] / / P_{p_{b}}} d_{p_{l}}[2] \neq\left(\mathscr{T}_{1} u_{2}, \mathscr{T}_{1} y_{2}\right) .
$$

This can be seen by choosing

$$
d_{p_{b}}[2]=\left(\left(\begin{array}{l}
0 \\
B
\end{array}\right)\left(\begin{array}{l}
0 \\
0
\end{array}\right)\left(\begin{array}{c}
\times \\
y_{0}^{p_{b}}(2)
\end{array}\right)\right) .
$$

In this case we have $y_{1}^{p_{b}}(2)=b B-B$. With $y_{2}^{p_{b}}(2)=B-l B$ from above we would have to choose

$$
y_{0}^{p_{b}}(2)=b B-l B \neq 0, \forall b \neq l
$$

to be consistent with $\left(\mathscr{T}_{1} u_{2}, \mathscr{T}_{2} y_{2}\right)$ and $P_{p_{b}}$. Therefore we can conclude that

$$
\left\|d_{p_{l}}[2]\right\|=B<\left\|d_{p_{b}}[2]\right\|,
$$

Since this holds for all $1 \leq b<l$, it follows that $q(2)=l$. It is trivial to verify that the remaining entries in table $I$ are consistent with equations (2.1),(2.2) for $P=P_{1}, C=C\left[\mathcal{P}_{l}\right]$ 
which establishes the claim, and completes the first part of the proof.

We now show that this leads to the unbounded increase of the gain of the closed loop operator as $l$ increases.

From the definition of $\Pi_{P / / C\left[\mathcal{P}_{l}\right]}$ we have

$$
\begin{aligned}
\| \Pi_{P / / C\left[\mathcal{P}_{l}\right] \|} & =\sup _{w_{0} \in \mathcal{W} \backslash\{0\}} \frac{\left\|\Pi_{P / / C\left[\mathcal{P}_{l}\right]}\left(w_{0}\right)\right\|}{\left\|w_{0}\right\|} \\
& \geq \frac{\left\|w_{1}\right\|}{\left\|w_{0}\right\|} \geq \frac{\left|y_{1}(3)\right|}{\left\|w_{0}\right\|} \\
& =\frac{B\left|\left(l-l^{2}\right)\right|}{B\|1,1-l, 0,0, \cdots\|} .
\end{aligned}
$$

Furthermore there exist scalars $L>1, \alpha, \beta>0$ such that

$$
\left|l-l^{2}\right| \geq \alpha l^{2}, \forall l \geq L
$$

and

$$
\|1,1-l, 0,0, \cdots\| \leq \beta l, \forall l \geq L
$$

Therefore

$$
\left\|\Pi_{P / / C\left[\mathcal{P}_{l}\right]}\right\| \geq \frac{\alpha}{\beta} l, \forall l \geq L
$$

as required.

\section{AN ADAPTIVE ALGORITHM WHICH DOES SCALE}

We now consider a version of the algorithm with an explicit non constant plant generating operator. Since we have shown that the availability of too many controllers right after initialization can lead to high gains, this behaviour is suppressed by limiting the number of available controllers over time. We observe that a similar procedure has been investigated in a stochastic context in [1], but with a rather different motivation. In particular we choose to relate the availability of controllers at time $k \in \mathbb{N}$ to the size of the observed signal $\left\|\mathscr{T}_{k} w_{2}\right\|$.

For this purpose we generalize the bound obtained in [6] to time varying plant sets.

Given an observation $w_{2} \in \mathcal{W}_{e}$ let the time $k_{*} \in \mathbb{N} \cup \infty$ when the parameter set $p_{*}$, corresponding to the unknown true plant $P_{p_{*}}$, is in the set of available plants for the first time, be given by

$$
k_{*}=\left\{\begin{aligned}
& \min \left\{k \in \mathbb{N} \mid p_{*} \in G\left(w_{2}\right)(k)\right\} \\
& \text { if } \exists k \text { s.t. } p_{*} \in G\left(y_{2}, y_{2}\right)(k) \\
& \infty \text { if not }
\end{aligned}\right.
$$

Theorem 4.1: Let the class of plants under consideration be given by $\mathcal{P}$ in equation (2.3), and let $p_{*} \in \mathcal{P}$. Let the plants $P_{p}, p \in \mathcal{P}$ and controllers $C_{p}, p \in \mathcal{P}$ be defined by equations (2.5)-(2.7) and (2.8) respectively. Let $S$ be defined by equations (2.9)-(2.19) and fullfill assumption 2.1. Let the switching controller $C$ be defined by equation (2.20). Suppose $\left\|\mathscr{T}_{k_{*}-1} w_{2}\right\| \leq \beta \in \mathbb{R}$ with $k_{*}$ defined by equation (4.30) and suppose $k_{*}<\infty$. Then there exists a function $\gamma: \Omega \rightarrow \mathbb{R}$ such that

$$
\left\|\mathscr{T}_{k} w_{2}\right\| \leq \gamma\left(G\left(w_{2}\right)(k)\right)\left(\beta+\left\|w_{0}\right\|\right), \forall k \in \mathbb{N} .
$$

Proof We only sketch this rather lengthy proof at this point since it follows in large parts directly from [6]. It is assumed that there exist a bound $\beta \in \mathbb{R}$ such that $\left\|\mathscr{T}_{k_{*}-1} w_{2}\right\| \leq \beta \in \mathbb{R}$. Therefore we perform the analysis along the lines of [6] from time $k=k_{*}$ onwards since by definition $p_{*} \in G\left(w_{2}\right)(k), k \geq k_{*}$ and the analysis of [6] is largely applicable. This leads to the desired result.

As a corollary to this result it can be shown that for $p_{*} \in G\left(w_{2}\right)=$ const. we obtain the bound given in [6]:

$$
\left\|w_{2}\right\| \leq \gamma\left\|w_{0}\right\|
$$

where

$$
\gamma=\gamma\left(G\left(w_{2}\right)(k)\right)=\text { const. }<\infty .
$$

Now let $\gamma: \Omega \rightarrow \mathbb{R}$ be the achievable gain bound in theorem 4.1. It is important to remark that the proof is constructive: an explicit expression for $\gamma$ can be given.

Let $\hat{\gamma}: \Omega \rightarrow \mathbb{R}$ be an increasing function with the property

$$
\hat{\gamma}(\mathcal{Q}) \geq \gamma(\mathcal{Q}), \forall \mathcal{Q} \in \Omega
$$

Let $v>2$. We now explicitly define a causal plant generating operator

$$
G: \mathcal{W}_{e} \rightarrow \operatorname{map}(\mathbb{N}, \Omega)
$$

by

$$
G\left(w_{2}\right)(k)=\mathcal{P}_{i(k)}
$$

where

$$
i(k)=\left\{\begin{array}{ll}
\max \{a \in \mathbb{N} \mid & \\
\left.\hat{\gamma}^{v}\left(\mathcal{P}_{a}\right)-\hat{\gamma}^{v}\left(\mathcal{P}_{1}\right) \leq\left\|\mathscr{T}_{k} w_{2}\right\|\right\} & \text { if } k<\infty \\
\infty & \text { if } k=\infty
\end{array}\right\} .
$$

We will now show that this modification of the switching algorithm gives us a new bound on $\left\|w_{2}\right\|$ where the algorithm automatically determines $\mathcal{P}_{i(k)}$.

Theorem 4.2: Let the plants sets under consideration be given by $\mathcal{P}$ in equation (2.3), and let $p_{*} \in \mathcal{P}$. Let the plants $P_{p}, p \in \mathcal{P}$ and controllers $C_{p}, p \in \mathcal{P}$ be defined by equations (2.5)-(2.7) and (2.8) respectively. Let $v>2$ and let $G$ be given by equations (4.33),(4.34). Let $S$ be defined by equations (2.9)-(2.19) and fullfill assumption 2.1 . Let $C$ be defined by equation (2.20) and consider the closed loop $\left[P_{p_{*}}, C\right]$ defined by equations (2.1),(2.2). Then for all $w_{0} \in \mathcal{W}$,

$$
\left\|w_{2}\right\| \leq \gamma_{\bmod }\left(\left\|w_{0}\right\|\right)
$$

where $\gamma_{\text {mod }}: \mathbb{R}_{+} \rightarrow \mathbb{R}_{+}$is given by

$$
\gamma_{\text {mod }}(r)=\beta_{1}+\beta_{2} r+\beta_{3} r^{2}
$$

with

$$
\begin{aligned}
\beta_{1} & =\hat{\gamma}^{v+2}\left(\mathcal{P}_{N}\right)+\hat{\gamma}\left(\mathcal{P}_{N}\right) \hat{\gamma}^{v}\left(\mathcal{P}_{1}\right) \\
\beta_{2} & =2 \hat{\gamma}^{2}\left(\mathcal{P}_{N}\right)+\hat{\gamma}^{1-v}\left(\mathcal{P}_{N}\right) \hat{\gamma}^{v}\left(\mathcal{P}_{1}\right) \\
\beta_{3} & =\hat{\gamma}^{2-v}\left(\mathcal{P}_{N}\right)
\end{aligned}
$$

and

$$
N:=\min \left\{i \geq 1 \mid p_{*} \in \mathcal{P}_{i}\right\} .
$$

Proof Let $w_{0} \in \mathcal{W}$ and let $k_{*}$ be given by equation (4.30). By equation (4.34) we have

$$
\left\|\mathscr{T}_{k} w_{2}\right\| \leq \hat{\gamma}^{v}\left(\mathcal{P}_{i(k)+1}\right) \forall k \in \mathbb{N} .
$$


By the fact that

$$
i\left(k_{*}\right) \geq N \geq i\left(k_{*}-1\right)+1
$$

which follows from the definition of $k_{*}$ and since $\hat{\gamma}$ is an increasing function we can write equation (4.35) with $k=k_{*}-1$ as

$$
\left\|\mathscr{T}_{k_{*}-1} w_{2}\right\| \leq \hat{\gamma}^{v}\left(\mathcal{P}_{i\left(k_{*}-1\right)+1}\right) \leq \hat{\gamma}^{v}\left(\mathcal{P}_{N}\right) .
$$

We now have to consider the two possibilities that

1) $k_{*}=\infty$

2) $k_{*}<\infty$.

In case 1 , since

$$
\hat{\gamma}\left(\mathcal{P}_{N}\right) \geq \gamma\left(\mathcal{P}_{N}\right) \geq 1
$$

it follows that

$$
\beta_{1} \geq \hat{\gamma}^{v+2}\left(\mathcal{P}_{N}\right) \geq \hat{\gamma}^{v}\left(\mathcal{P}_{N}\right)
$$

hence

$$
\left\|w_{2}\right\|=\left\|\mathscr{T}_{k_{*}-1} w_{2}\right\| \leq \hat{\gamma}^{v}\left(\mathcal{P}_{N}\right) \leq \beta_{1} .
$$

In case 2 with $k \leq k_{*}-1$ it follows similarly to 1 that

$$
\left\|\mathscr{T}_{k} w_{2}\right\| \leq \beta_{1} \text {. }
$$

For $k>k_{*}-1$ we have by equation (4.34), Theorem 4.1 and equations (4.31),(4.37) that

$$
\begin{aligned}
\hat{\gamma}^{v}\left(\mathcal{P}_{i(k)}\right) & \leq\left\|\mathscr{T}_{k} w_{2}\right\|+\hat{\gamma}^{v}\left(\mathcal{P}_{1}\right) \\
& \leq \gamma\left(\mathcal{P}_{i(k)}\right)\left(\left\|\mathscr{T}_{k_{*}-1} w_{2}\right\|+\left\|w_{0}\right\|\right)+\hat{\gamma}^{v}\left(\mathcal{P}_{1}\right) \\
& \leq \hat{\gamma}\left(\mathcal{P}_{i(k)}\right)\left(\hat{\gamma}^{v}\left(\mathcal{P}_{N}\right)+\left\|w_{0}\right\|\right)+\hat{\gamma}^{v}\left(\mathcal{P}_{1}\right) .
\end{aligned}
$$

Multiplication with $\hat{\gamma}^{1-v}\left(\mathcal{P}_{i(k)}\right)>0$ yields

$\hat{\gamma}\left(\mathcal{P}_{i(k)}\right) \leq \hat{\gamma}^{2-v}\left(\mathcal{P}_{i(k)}\right)\left(\left\|w_{0}\right\|+\hat{\gamma}^{v}\left(\mathcal{P}_{N}\right)\right)+\hat{\gamma}^{1-v}\left(\mathcal{P}_{i(k)}\right) \hat{\gamma}^{v}\left(\mathcal{P}_{1}\right)$.

Furthermore since by equations (4.33),(4.34) $G$ satisfies equation (2.12), that is that

$$
G\left(w_{2}\right)(k) \subseteq G\left(w_{2}\right)(l), \forall l \geq k,
$$

by equation (4.36) we have $\mathcal{P}_{N} \subseteq \mathcal{P}_{i(k)}$. Since $\hat{\gamma}$ is increasing it follows that

$$
\hat{\gamma}^{q-v}\left(\mathcal{P}_{i(k)}\right) \leq \hat{\gamma}^{q-v}\left(\mathcal{P}_{N}\right), \forall q<v
$$

and hence we obtain

$$
\hat{\gamma}\left(\mathcal{P}_{i(k)}\right) \leq \hat{\gamma}^{2}\left(\mathcal{P}_{N}\right)+\hat{\gamma}^{2-v}\left(\mathcal{P}_{N}\right)\left\|w_{0}\right\|+\hat{\gamma}^{1-v}\left(\mathcal{P}_{N}\right) \hat{\gamma}^{v}\left(\mathcal{P}_{1}\right) .
$$

Hence by Theorem 4.1,

$$
\begin{aligned}
\left\|\mathscr{T}_{k} w_{2}\right\| \leq & \gamma\left(\mathcal{P}_{i(k)}\right)\left(\left\|\mathscr{T}_{k_{*}-1} w_{2}\right\|+\left\|w_{0}\right\|\right) \\
\leq & \left(\hat{\gamma}^{v}\left(\mathcal{P}_{N}\right)+\left\|w_{0}\right\|\right) \\
& {\left[\hat{\gamma}^{2}\left(\mathcal{P}_{N}\right)+\hat{\gamma}^{2-v}\left(\mathcal{P}_{N}\right)\left\|w_{0}\right\|+\hat{\gamma}^{1-v}\left(\mathcal{P}_{N}\right) \hat{\gamma}^{v}\left(\mathcal{P}_{1}\right)\right] } \\
\leq & \beta_{1}+\beta_{2}\left\|w_{0}\right\|+\beta_{3}\left\|w_{0}\right\|^{2}, \forall k \in \mathbb{N} .
\end{aligned}
$$

We observe that the bound is independent of $k$ and therefore

$$
\left\|w_{2}\right\| \leq \beta_{1}+\beta_{2}\left\|w_{0}\right\|+\beta_{3}\left\|w_{0}\right\|^{2}
$$

as required.
We remark that there is no requirement of finiteness of the "limit set" $\lim _{i \rightarrow \infty} \mathcal{P}_{i} \subset \mathcal{P}$, for example, this result can be applied to the plant sets $\mathcal{P}_{l}$ of Section 3 . In these cases, the controller can be used in a universal context: if there is no knowledge of $l>0$, then the switching controller realized achieves the bound of Theorem 4.2 with an implementation that never requires the consideration of more than a finite number of plant estimators at any given time.

\section{CONCLUSiON}

We have shown by an example that MMAC suffers from a poor degradation of the closed loop induced gain under certain scalings of the size of the uncertainty (for example the number of candidate plants).

By replacing the notion of an induced gain by that of a quadratic gain function, BIBO stability results were obtained where the resulting bounds are independent of the maximum size of the parametric uncertainty, (but are dependent only on the 'true' plant).

Topics of current research include the generalization of the result to a wider class of plants and controllers as well as the improvement of the bounds $\gamma$.

\section{REFERENCES}

[1] Marco Campi, João Pedro Hespanha, and Maria Prandini. Cautious hierarchical switching control of stochastic linear systems. Int. J. of Adaptive Contr. and Signal Processing, Special Issue on New Approaches to Adaptive Control, 18:319-333, May 2004.

[2] T. P. Fisher-Jeffes. Multiple-Model Switching Control to Achieve Asymptotic Robust Performance. PhD thesis, University of Cambridge, 2003.

[3] M. French. Smooth adaptive controllers have discontinuous closed loop operators. Leuven: Sixteenth International Symposium on Mathematical Theory of Networks and Systems (MTNS2004), 2004.

[4] M. French. Adaptive control and robustness in the gap metric. IEEE Transactions on Automatic Control, to appear Feb. 2008.

[5] M. French, A. Ilchmann, and E. P. Ryan. Robustness in the graph topolgy of a common adaptive controller. SIAM Journal of Control and Optimization, 45(5):1736-1757, 2006.

[6] M. French and S. Trenn. $l^{p}$ gain bounds for switched adaptive controllers. In Proc. of the 44th IEEE Conf. on Decision and Control and European Control Conf. ECC 2005, Seville (Spain), pages 28652870, 2005.

[7] T. T. Georgiou and M. C. Smith. Robustness analysis of nonlinear feedback systems: An input-output approach. IEEE Transactions on Automatic Control, 42(9):1200-1220, September 1997.

[8] A. Morse. Supervisory control of families of linear set-point controllers - part 1: Exact matching. IEEE Transactions on Automatic Control, 41(10):1413-1431, 81996.

[9] A. Morse. Supervisory control of families of linear set-point controllers - part 2: Robustness. IEEE Transactions on Automatic Control, 42(9):1200-1220, September 1997.

[10] G. Vinnicombe. Examples and counterexamples in finite $l_{2}$-gain adaptive control. Leuven: Sixteenth International Symposium on Mathematical Theory of Networks and Systems (MTNS2004), 2004. 